\title{
Chiral surface waves for enhanced circular dichroism
}

\author{
Giovanni Pellegrini, ${ }^{*}$ Marco Finazzi, Michele Celebrano, Lamberto Duò, and Paolo Biagioni ${ }^{\dagger}$ \\ Dipartimento di Fisica, Politecnico di Milano, Piazza Leonardo da Vinci 32, 20133 Milano, Italy \\ (Received 18 November 2016; revised manuscript received 16 May 2017; published 6 June 2017)
}

\begin{abstract}
We present a novel chiral sensing platform that combines a one-dimensional photonic crystal design with a birefringent surface defect. The platform sustains simultaneous transverse electric and transverse magnetic surface modes, which are exploited to generate chiral surface waves. The present design provides homogeneous and superchiral fields of both handednesses over arbitrarily large areas in a wide spectral range, resulting in the enhancement of the circular dichroism signal by more than two orders of magnitude, thus paving the road toward the successful combination of surface-enhanced spectroscopies and electromagnetic superchirality.
\end{abstract}

DOI: 10.1103/PhysRevB.95.241402

Chiral objects are three-dimensional bodies characterized by being geometrically distinct from their mirror image. Objects of opposite chirality, called enantiomers, share most of their physical properties and their chiral nature emerges only when they interact with a chiral environment. This geometrical feature, which plays a crucial role in many biochemical processes, is ubiquitous in biomolecules, the most outstanding example being DNA [1,2]. As a consequence, the analysis and separation of chiral molecules has lately gained increasing importance in the pharmaceutical and biochemical industry. Circular dichroism (CD) spectroscopy, i.e., the measurement of the differential absorption between left (L) and right (R) circularly polarized light (CPL), is one of the most relevant tools for enantiomer discrimination. However, CD signals are usually extremely weak if compared to those of standard achiral absorption spectroscopies, which makes the analysis of small amounts of chiral analytes extremely challenging [3-6].

In this context, novel approaches have been proposed over the last few years to enhance the CD signal by tailoring the chiral properties of the probing electromagnetic field [5-9] through the control of the associated optical chirality $C$ [5], which is defined as

$$
C=-\frac{\varepsilon_{0} \omega}{2} \operatorname{Im}\left(\mathbf{E}^{*} \cdot \mathbf{B}\right) .
$$

This quantity is a time-even pseudoscalar, i.e., it behaves as a chiral object under mirror inversion, and determines the degree of asymmetry in the absorption rate of a chiral molecule between $\mathrm{L}$ and $\mathrm{R}$ circularly polarized light in the dipolar approximation. Circularly polarized plane waves are a common example of a chiral electromagnetic field, with their optical chirality for a unitary intensity wave expressed as $C_{\mathrm{CPL}}^{\mathrm{L}, \mathrm{R}}= \pm \varepsilon_{0} \omega / 2$ [5]. Likewise, a field distribution where $C$ is beyond the $C_{\mathrm{CPL}}^{\mathrm{L}, \mathrm{R}}$ value is dubbed "superchiral."

In this framework, an ideal chiral sensing platform should be able to (i) provide uniform superchiral optical fields $\left(|C|>\left|C_{\mathrm{CPL}}^{\mathrm{L}, \mathrm{R}}\right|\right)$ over large areas, (ii) generate optical chiralities of both handednesses upon reversing the polarization state of the incident field, and (iii) work at wavelengths ranging from the near-UV to the IR depending on the platform design, with

\footnotetext{
*giovanni.pellegrini@polimi.it

†paolo.biagioni@polimi.it
}

a particular attention to the high energy range of the spectrum, below $400 \mathrm{~nm}$, where most electronic molecular transitions occur [9-11].

Plasmonic nanostructures have been widely proposed and employed as a viable solution for the enhancement of the optical chiral response of biomolecules [4,7-22]. However, despite the recent results, to date chiral plasmonic sensing does not meet all the above criteria simultaneously. In particular, superchiral optical fields are spatially confined to the socalled plasmonic "hot spots" and large homogeneous optical chiralities are usually obtained only for chiral plasmonic nanostructures, thus hindering the possibility of handedness switching upon reversal of the incident polarization state. Indeed, the utmost importance of molecular spectroscopy in the blue and near-UV energy range, which is not accessible with standard Au plasmonics, is pushing for the adoption of novel plasmonic materials such as aluminum, which can operate in the blue and near-UV range [23].

Uniform surface-enhanced fields for sensing, which are not found in substrates based on nanoparticles, are instead obtained when surface plasmon polaritons or Bloch surface waves are employed [24-26]. However, surface plasmon polaritons, which are propagating longitudinal oscillations of the electron plasma at the surface of a metal, are intrinsically transverse magnetic (TM) surface modes, therefore generating local fields that carry no optical chirality, in analogy with linearly polarized plane waves. Bloch surface waves, on the other side, are surface waves created by a semi-infinite one-dimensional photonic crystal (1DPC), usually terminated with a surface defect, and support both transverse electric (TE) and TM modes. However, the two dispersion relations of such modes do not overlap in ordinary conditions and therefore the TE and TM waves cannot be excited simultaneously, again preventing the creation of chiral surface waves (CSWs).

To address all these issues, we introduce a novel chiral sensing platform based on the combination of a 1DPC with a properly engineered anisotropic surface defect. Electrodynamics calculations show that such a platform, which is well within the modern fabrication capabilities, supports CSWs originating from the coherent superposition of TE and TM surface modes, providing (i) homogeneous and (ii) switchable superchiral fields over arbitrarily large areas and wide spectral ranges. Additionally, (iii) the use of standard dielectric materials allows moving the operation wavelength toward the high-energy blue end of the spectrum. The design 


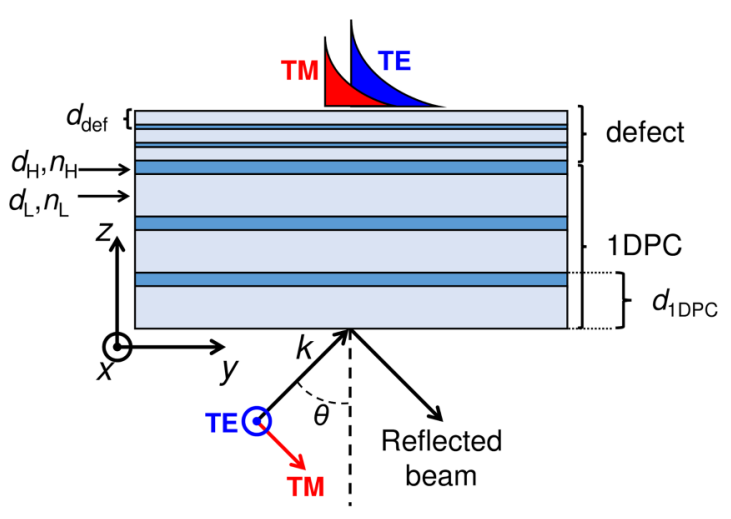

FIG. 1. A schematic representation of the 1DPC sensing platform, with the incoming TE and TM linearly polarized waves represented at the bottom, and the corresponding TE and TM surface modes at the top. Crystal and defect periods are defined as $d_{1 \mathrm{DPC}}$ and $d_{\mathrm{def}}$, respectively, while $d_{\mathrm{H}, \mathrm{L}}$ stands for the thickness of the high and low refractive index materials in the 1DPC.

of the chiral sensing platform is based on two fundamental considerations regarding the optical properties of a semiinfinite 1DPC terminated with a surface defect. Specifically, (i) 1DPCs support both TE and TM surface modes, and (ii) the slopes of the respective dispersion relations are a function of the effective refractive index of the defect [24,27]. It follows naturally that, for a properly designed structure terminated with an optically anisotropic defect, the TE and TM dispersion relations can superimpose in the $\left(k_{\|}, \omega\right)$ space within the band structure forbidden region, where $k_{\|}$is the wave vector component parallel to the crystal surface. This eventually enables the coherent excitation of both TE and TM modes in a wide spectral range, thus obtaining a chiral surface wave upon the introduction of a $\pi / 2$ phase shift between them.

A practical implementation of the proposed design is illustrated in Fig. 1. The semi-infinite 1DPC consists of alternating high $\left(n_{\mathrm{H}}\right)$ and low $\left(n_{\mathrm{L}}\right)$ refractive index materials. For our structure we choose $\mathrm{Ta}_{2} \mathrm{O}_{5}\left(n_{\mathrm{H}}=2.06+0.001 i\right)$ and $\mathrm{SiO}_{2}\left(n_{\mathrm{L}}=1.454+0.0001 i\right)$, which are a standard choice for 1DPC fabrication, and allow for the generation of both TE and TM surface modes [28-30]. Material dispersion is ignored for simplicity, yet it can be straightforwardly included in the design process if needed. The 1DPC is then terminated with an optically anisotropic defect characterized by the diagonal dielectric tensor:

$$
\overline{\bar{\varepsilon}}=\left(\begin{array}{ccc}
\varepsilon_{\mathrm{xx}} & 0 & 0 \\
0 & \varepsilon_{\mathrm{xx}} & 0 \\
0 & 0 & \varepsilon_{\mathrm{zz}}
\end{array}\right) .
$$

In order to obtain the necessary uniaxial birefringence, we exploit form anisotropy and design the defect as an additional $\mathrm{Ta}_{2} \mathrm{O}_{5} / \mathrm{SiO}_{2}$ multilayer with a period $d_{\text {def }}$ much smaller than the principal period $d_{1 \mathrm{DPC}}$. The diagonal elements of the tensor are then obtained by standard Maxwell homogenization and expressed as [31]

$$
\begin{aligned}
& \varepsilon_{\mathrm{xx}}=f_{\text {def }} \varepsilon_{\mathrm{H}}+\left(1-f_{\mathrm{def}}\right) \varepsilon_{\mathrm{L}}, \\
& \varepsilon_{\mathrm{zZ}}=\left[\frac{f_{\mathrm{def}}}{\varepsilon_{\mathrm{H}}}+\frac{\left(1-f_{\mathrm{def}}\right)}{\varepsilon_{\mathrm{L}}}\right]^{-1},
\end{aligned}
$$
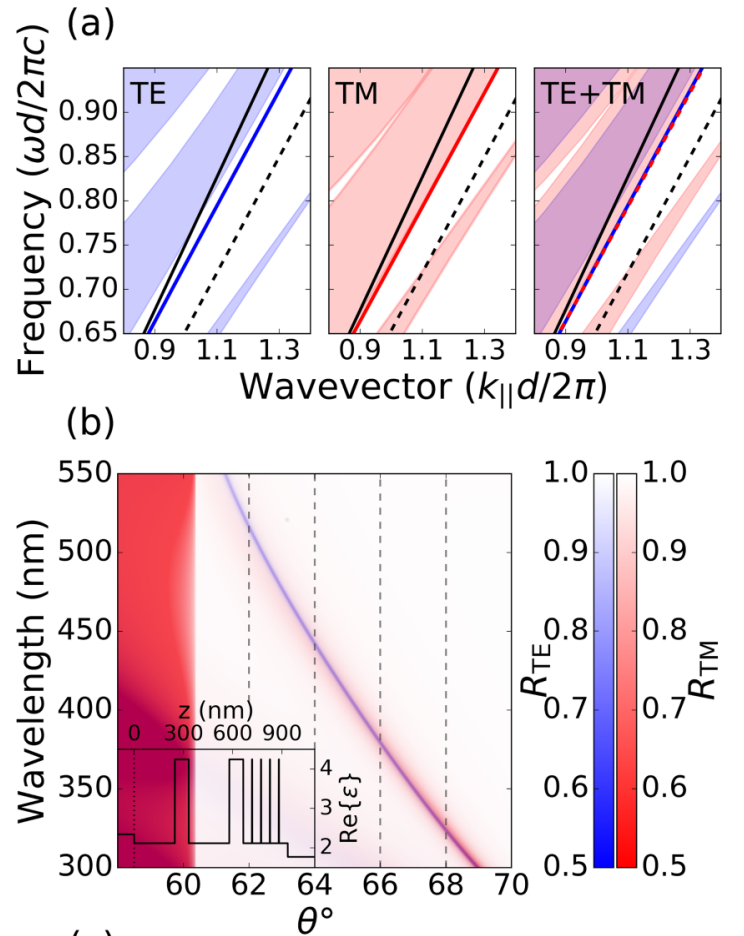

(c)

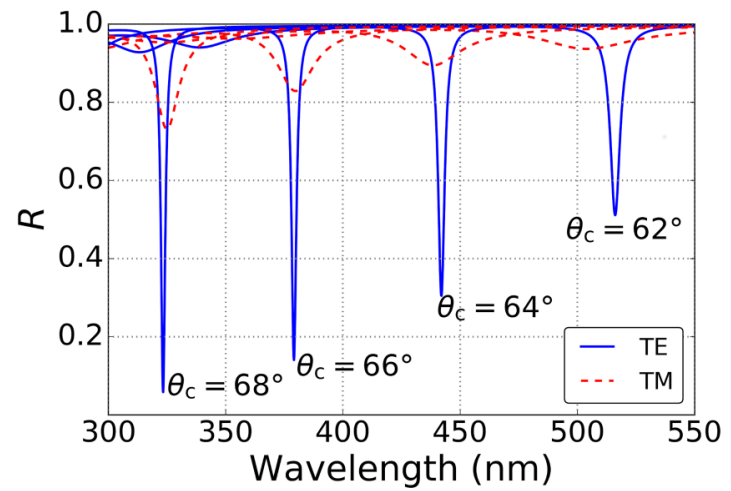

FIG. 2. (a) 1DPC band structures for TE and TM illumination, and their superposition. The black continuous and dashed lines represent the light line in water and in the $n_{\text {inc }}=1.53$ incident medium, respectively. (b) Superimposed reflection maps for TE and TM illuminations. The thin dashed lines indicate a spectral slice taken at constant incident angle $\theta_{c}$. Inset: $z$ profile of the 1DPC dielectric constants. (c) Spectral slices of the reflectivity map for TE and TM illuminations at different incident angles $\theta_{\mathrm{c}}$.

where $\varepsilon_{\mathrm{H}, \mathrm{L}}=n_{\mathrm{H}, \mathrm{L}}^{2}, f_{\text {def }}$ is the filling factor of the high refractive index material, and the upper surface of the defect is in contact with water. All the geometrical parameters of the 1DPC are detailed in the Supplemental Material (SM) [32].

The TE and TM band structure for the semi-infinite 1DPC, calculated with the MIT Photonic-Bands package adopting the supercell method [33], are reported in Fig. 2(a). The 1DPC supports both TE and TM surface modes, highlighted by the lines within the forbidden regions of the band structure (white areas). The shaded regions correspond to allowed optical modes extended inside the 1DPC. The same modes can either be extended or exponentially decaying in the upper semispace depending on their position above or below the light line 
$\omega=v k_{\|}$, where $v=c / n_{\text {water }}$ is the speed of light in water and $n_{\text {water }}=1.33$. By superimposing the two band structures, as displayed in the right panel of Fig. 2(a), the optically anisotropic defect allows us to obtain nicely overlapping TE and TM surface mode dispersion relations in the $\left(k_{\|}, \omega\right)$ space. This achievement offers the unique possibility to launch surface modes with arbitrary polarization states in a wide spectral range, in direct contrast to both standard surface plasmon polaritons and isolated Bloch surface waves.

We model the same phenomenon for an actual 1DPC with a finite number of periods, illuminated in Kretschmann configuration for proper momentum matching. We illuminate the finite 1DPC, consisting of two crystal periods plus the anisotropic surface defect, from an incident medium with refractive index $n_{\text {inc }}=1.53$. The reflection maps for both TE and TM illuminations in Fig. 2(b) display the surface modes as a narrow dark band (TE mode), superimposed to a lighter halo (TM mode), in agreement with the overlapping observed for the semi-infinite 1DPC, demonstrating how the adopted design approach is sound even for realistic finite size structures. The total internal reflection onset is clearly visible around the $\theta \simeq 60^{\circ}$ incident angle as a sharp variation in the reflected intensity.

The mode overlapping is also clearly illustrated in Fig. 2(c), which reports spectral slices of the reflectivity maps computed at several coupling angles $\theta_{c}$ [dashed lines in Fig. 2(b)]. The modes appear as well superimposed reflectivity dips at coupling wavelengths $\lambda_{\mathrm{c}}$ ranging from 300 to $550 \mathrm{~nm}$. In this framework, the maximum optical chirality $C$ is obtained by illuminating the sample at $\left(\lambda_{\mathrm{c}}, \theta_{\mathrm{c}}\right)$ with $\mathrm{L}$ or $\mathrm{R}$ elliptically polarized light. The dephasing between the TE and TM incident plane wave components is thus defined as $\phi_{\mathrm{c}}=$ $\pi / 2-\Delta \phi_{\mathrm{TE}, \mathrm{TM}}$, where $\Delta \phi_{\mathrm{TE}, \mathrm{TM}}$ is the relative phase delay introduced by the 1DPC.

Figure 3 reports the optical chirality map calculated $5 \mathrm{~nm}$ above the 1DPC surface, reminding that along the $z$ axis we have an $e^{-k_{z} z}$ exponential decay with $k_{z}=\sqrt{k_{\|}^{2}-k^{2}}$. We adopt a unitary intensity $L$ elliptical illumination and normalize the results to the modulus of the optical chirality for a circularly

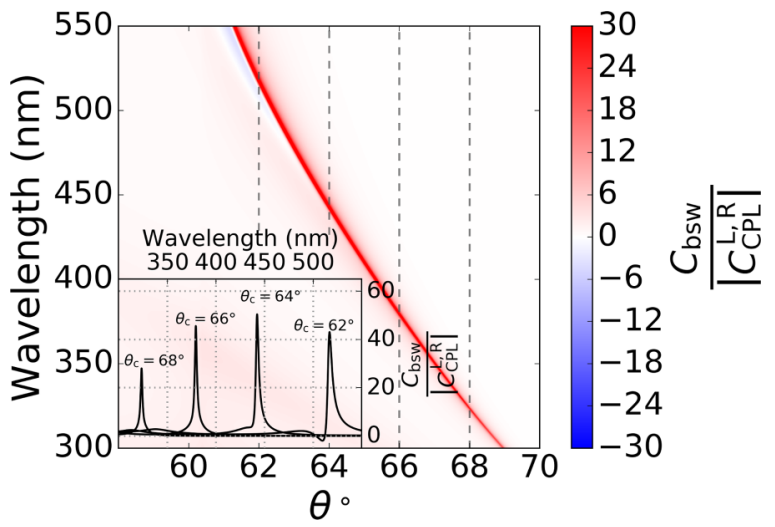

FIG. 3. Optical chirality enhancement map calculated at the coupling phase $\phi_{\mathrm{c}}, 5 \mathrm{~nm}$ above the 1DPC surface. The thin dashed lines indicate a spectral slice taken at constant incident angle $\theta_{\mathrm{c}}$. Inset: Optical chirality enhancement spectral slices taken at different incident angles $\theta_{\mathrm{c}}$. polarized plane wave $\left|C_{\mathrm{CPL}}^{\mathrm{L}, \mathrm{R}}\right|$. The coherent superposition of the TE and TM surface modes with the appropriate coupling phase $\phi_{\mathrm{c}}$ results in a sharp optical chirality enhancement well above one order of magnitude in the whole analyzed spectral range. Superchirality is only achieved if both TE and TM surface modes are simultaneously launched, while the mere excitation of TE and TM evanescent waves is not sufficient to generate sizable superchirality.

The obtained superchiral field meets all the criteria for an ideal sensing platform. First, the in-plane translational invariance of the system implies that the chirality enhancement is obtained over arbitrarily large areas, where the only realistic constraints are imposed by limitations in the illumination and fabrication processes. Second, the handedness of the optical chirality can be readily switched by alternating between $\mathrm{L}$ and $\mathrm{R}$ incident elliptical polarization states. Third, as illustrated in Fig. 3, the platform can operate in a wide spectral range in the blue and near-UV end of the spectrum, opening up the possibility to perform surface enhanced CD spectroscopy. Finally, it is worth stressing that the obtained surface wave is intrinsically chiral, i.e., the optical chirality does not originate from the interference between the incident and local fields, which is often the case for most plasmonic platforms [9].

To further investigate this design, we perform $\mathrm{CD}$ numerical simulations by adding a thin film of a randomly oriented chiral analyte on top of the 1DPC. To this end we solve the multilayer transfer matrix problem including the constitutive relations for the chiral medium [34]:

$$
\begin{aligned}
& \mathbf{D}=\varepsilon_{\mathrm{c}} \varepsilon_{0} \mathbf{E}+i \xi_{\mathrm{c}} \mathbf{B}, \\
& \mathbf{H}=\frac{\mathbf{B}}{\mu \mu 0}+i \xi_{\mathrm{c}} \mathbf{E},
\end{aligned}
$$

where $\varepsilon_{\mathrm{c}}$ and $\mu$ denote the relative permittivity and permeability, and $\xi_{\mathrm{c}}$ is the chiral optical admittance, i.e., the parameter that controls the chirality strength [34]. We work with a nonmagnetic medium $(\mu=1)$, and we define the optical parameters of the chiral medium as [13]

$$
\begin{gathered}
\varepsilon_{\mathrm{c}}=\varepsilon_{\mathrm{c} 0}-\gamma_{\mathrm{c}}\left(\frac{1}{\hbar \omega-\hbar \omega_{0}+i \Gamma}-\frac{1}{\hbar \omega+\hbar \omega_{0}+i \Gamma}\right), \\
\xi_{\mathrm{c}}=\beta_{\mathrm{c}}\left(\frac{1}{\hbar \omega+\hbar \omega_{0}+i \Gamma}+\frac{1}{\hbar \omega-\hbar \omega_{0}+i \Gamma}\right),
\end{gathered}
$$

where the coefficients $\gamma_{\mathrm{c}}$ and $\beta_{\mathrm{c}}$ determine the magnitude of the absorptive and chiral properties. The energy of the molecular absorption resonance is $\omega_{0}=2 \pi c / \lambda_{0}$, with $\lambda_{0}=380 \mathrm{~nm}$, while $\gamma_{\mathrm{c}}=2.5 \times 10^{-3} \mathrm{eV}$ and $\beta_{\mathrm{c}}=2.5 \times 10^{-9} \mathrm{eV} / \Omega$ are chosen to match the optical properties of Flavin mononucleotide (FMN) [10]. Finally we set the damping and background optical constants as $\Gamma=0.1 \mathrm{eV}$ and $\varepsilon_{\mathrm{c} 0}=1.77$ [9].

In the following we define the plane-wave reference $\mathrm{CD}$ signal as $\mathrm{CD}_{\text {ref }}=A_{\text {ref }}^{\mathrm{R}}-A_{\text {ref }}^{\mathrm{L}}$, where $A_{\text {ref }}^{\mathrm{R}, \mathrm{L}}=-\log \left(T^{\mathrm{R}, \mathrm{L}}\right)$ and the transmittance $T^{\mathrm{R}, \mathrm{L}}$ is computed by normally illuminating a chiral thin film, deposited on a $n=1.45$ glass substrate, with circularly polarized light of unitary intensity. On the other hand, we define the surface wave $C D$ signal as $\mathrm{CD}_{\mathrm{CSW}}=A_{\mathrm{CSW}}^{\mathrm{R}}-A_{\mathrm{CSW}}^{\mathrm{L}}$. In this case we use an equivalent absorbance defined as $A_{\mathrm{CSW}}^{\mathrm{R}, \mathrm{L}}=-\log \left(R^{\mathrm{R}, \mathrm{L}}\right)$, where the 


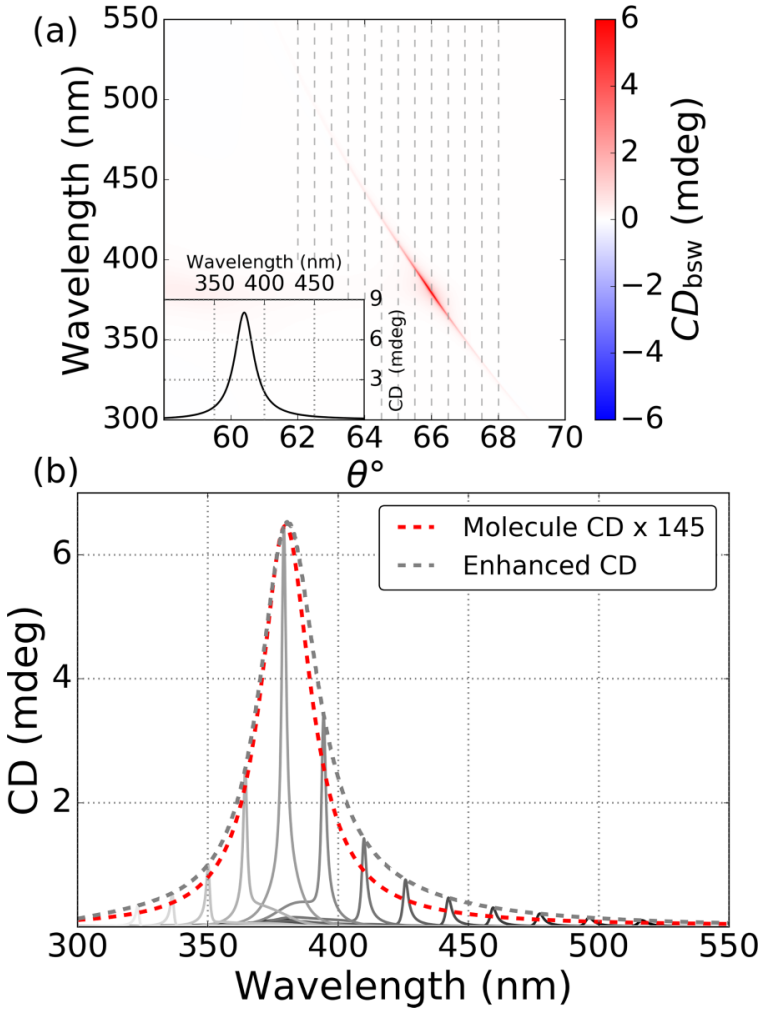

FIG. 4. (a) Chirality map for a $100 \mathrm{ng} / \mathrm{cm}^{2} \mathrm{FMN}$ film deposited on the 1DPC platform. The thin dashed lines indicate a spectral slice taken at constant incident angle $\theta_{c}$. Inset: CD spectrum for a $18 \mu \mathrm{g} / \mathrm{cm}^{2}$ reference FMN film deposited on a glass substrate. (b) Spectral slices taken at different incident angles $\theta_{c}$ (gray continuous lines), envelope curve (gray dashed line), and CD spectrum for a $100 \mathrm{ng} / \mathrm{cm}^{2}$ reference FMN film deposited on a glass substrate (red dashed line).

transmittance is substituted by the reflectivity $R^{\mathrm{R}, \mathrm{L}}$ in total internal reflection configuration, adopting $\mathrm{L}$ and $\mathrm{R}$ incident elliptical polarization states. The CD signal enhancement is finally defined as $C D_{\mathrm{Csw}} / C D_{\text {ref. }}$. The same quantities can be used to define the reference and surface wave dissymmetry factors as $g_{\text {ref }}=\frac{T^{\mathrm{R}}-T^{\mathrm{L}}}{T^{\mathrm{R}}+T^{\mathrm{L}}}$ and $g_{\mathrm{CSW}}=\frac{R^{\mathrm{R}}-R^{\mathrm{L}}}{R^{\mathrm{R}}+R^{\mathrm{L}}}$, respectively [5]. Likewise the dissymmetry enhancement can be written as $g_{\mathrm{CSW}} / g_{\text {ref }}$. In the following we shall only refer to the CD enhancement figure of merit, since it is possible to show that, for CD signal amplitudes that are typically very small $(\ll 1)$, $C D_{\mathrm{CSW}} / C D_{\text {ref }} \simeq g_{\mathrm{CSW}} / g_{\text {ref }}$.

Figure 4(a) reports the CD signal map for a $5 \mathrm{~nm}$ chiral film, corresponding to a FMN surface concentration of about $100 \mathrm{ng} / \mathrm{cm}^{2}$ [10]. The map reveals a strong CD signal perfectly aligned with the chiral surface mode. The maximum CD signal appears at the crossing between the molecule electronic transition [inset of Fig. 4(a)] and the chiral modes, reaching values up to $\mathrm{CD}_{\mathrm{CSW}} \simeq 6 \mathrm{mdeg}$ [35], with signal enhancements up to $\mathrm{CD}_{\mathrm{CSW}} / \mathrm{CD}_{\text {ref }} \simeq 150$. In a traditional transmittance setup the overall signal for the same molecular film would be well below the 0.1 mdeg level, whereas $1 \mathrm{mdeg}$ is considered the common limit of detection for standard CD spectrometers [9]. It follows that a very rough estimate of the limit of detection for FMN films measured with the 1DPC returns a minimum measurable surface concentration of the order of $15 \mathrm{ng} / \mathrm{cm}^{2}$. Therefore, we emphasize that, even in the case of extremely low concentrations $\left(\simeq 15 \mathrm{ng} / \mathrm{cm}^{2}\right)$, we can directly detect the absorption $\mathrm{CD}$ signal without recurring to fluorescence measurements, at variance with previous superchiral approaches $[5,6]$.

It is finally of the utmost importance to verify that the wide spectral range of the chiral sensing platforms allows for the reconstruction of $\mathrm{CD}$ spectral line shapes. This is simply accomplished by performing several $\mathrm{CD}$ spectral slices at different coupling angles $\theta_{\mathrm{c}}$ [dashed lines in Fig. 4(a)], and then calculating the corresponding envelope curve. Figure 4(b) demonstrates how the sensor provides CD signal enhancement above 2 orders of magnitude, and how the envelope curve procedure allows us to faithfully reconstruct the molecule $\mathrm{CD}$ line shape, thus laying the foundations for surface enhanced CD spectroscopy measurements. It is nevertheless worth noting that this approach introduces a minor overestimation of the transition linewidth, caused by a slight modulation in the sensor efficiency attributed to the molecule resonant absorption.

In conclusion, we have presented the design of a 1DPC sensing platform capable of supporting chiral surface waves generated by the coherent superposition of TE and TM surface modes. Chiral surface waves provide homogeneous, superchiral, and switchable local fields over arbitrarily large areas in a wide spectral range. The present design provides optical chirality enhancements well above 1 order of magnitude and CD signal enhancements of more than 2 orders of magnitude, allowing for the direct measurement of absorption CD signals down to surface concentrations of the order of $15 \mathrm{ng} / \mathrm{cm}^{2}$, hence paving the way to fluorescence-free surface enhanced CD sensing and spectroscopy on extremely low analyte amounts. The simple geometry makes the system an ideal candidate for automated design, optimization procedures, and straightforward fabrication with standard multilayer deposition techniques. Most importantly, the adopted geometry is ideal for the integration with lab-on-chip technologies and microfluidic networks on top of the surface of the device, allowing for the targeting of molecules in solutions in well controlled environments, extremely small volumes, and low concentrations. Finally, the planar geometry and the employed materials are also ideal for surface functionalization and for the analysis of iso-oriented chiral molecules, therefore allowing, as a notable example, for the analysis of the spectral signatures from tertiary and quaternary structures in proteins [21].

The research leading to these results has received funding from the Italian Ministry of Education, Universities and Research (MIUR) through the PRIN 2015 program (Project No. 2015FSHNCB "Plasmon-enhanced vibrational circular dichroism").
[1] U. Meierhenrich, Amino Acids and the
Asymmetry of Life 2008).
[2] G. D. Fasman, Circular Dichroism and the Conformational Analysis of Biomolecules (Springer Science + Business Media, New York, 1996). 
[3] L. D. Barron, Molecular Light Scattering and Optical Activity (Cambridge University Press, Cambridge, 2004).

[4] E. Hendry, T. Carpy, J. Johnston, M. Popland, R. V. Mikhaylovskiy, A. J. Lapthorn, S. M. Kelly, L. D. Barron, N. Gadegaard, and M. Kadodwala, Nat. Nanotechnol. 5, 783 (2010).

[5] Y. Tang and A. E. Cohen, Phys. Rev. Lett. 104, 163901 (2010).

[6] Y. Tang and A. E. Cohen, Science 332, 333 (2011).

[7] M. Schäferling, X. Yin, and H. Giessen, Opt. Express 20, 26326 (2012).

[8] M. Schäferling, X. Yin, N. Engheta, and H. Giessen, ACS Photon. 1, 530 (2014).

[9] M. L. Nesterov, X. Yin, M. Schäferling, H. Giessen, and T. Weiss, ACS Photon. 3, 578 (2016).

[10] N. A. Abdulrahman, Z. Fan, T. Tonooka, S. M. Kelly, N. Gadegaard, E. Hendry, A. O. Govorov, and M. Kadodwala, Nano Lett. 12, 977 (2012).

[11] M. Schäferling, N. Engheta, H. Giessen, and T. Weiss, ACS Photon. 3, 1076 (2016).

[12] A. O. Govorov, Z. Fan, P. Hernandez, J. M. Slocik, and R. R. Naik, Nano Lett. 10, 1374 (2010).

[13] A. O. Govorov and Z. Fan, Chem. Phys. Chem. 13, 2551 (2012).

[14] E. Hendry, R. V. Mikhaylovskiy, L. D. Barron, M. Kadodwala, and T. J. Davis, Nano Lett. 12, 3640 (2012).

[15] M. Schäferling, D. Dregely, M. Hentschel, and H. Giessen, Phys. Rev. X 2, 031010 (2012).

[16] V. K. Valev, J. J. Baumberg, C. Sibilia, and T. Verbiest, Adv. Mater. 25, 2509 (2013).

[17] F. Lu, Y. Tian, M. Liu, D. Su, H. Zhang, A. O. Govorov, and O. Gang, Nano Lett. 13, 3145 (2013).

[18] B. Frank, X. Yin, M. Schäferling, J. Zhao, S. M. Hein, P. V. Braun, and H. Giessen, ACS Nano 7, 6321 (2013).

[19] Y. Liu, R. Wang, and X. Zhang, Opt. Express 22, 4357 (2014).

[20] V. K. Valev, J. J. Baumberg, B. D. Clercq, N. Braz, X. Zheng, E. J. Osley, S. Vandendriessche, M. Hojeij, C. Blejean, J. Mertens, C. G. Biris, V. Volskiy, M. Ameloot, Y. Ekinci, G. A. E. Vandenbosch, P. A. Warburton, V. V. Moshchalkov, N. C. Panoiu, and T. Verbiest, Adv. Mater. 26, 4074 (2014).
[21] R. Tullius, A. S. Karimullah, M. Rodier, B. Fitzpatrick, N. Gadegaard, L. D. Barron, V. M. Rotello, G. Cooke, A. Lapthorn, and M. Kadodwala, J. Am. Chem. Soc. 137, 8380 (2015).

[22] M. Finazzi, P. Biagioni, M. Celebrano, and L. Duò, Phys. Rev. B 91, 195427 (2015).

[23] M. W. Knight, N. S. King, L. Liu, H. O. Everitt, P. Nordlander, and N. J. Halas, ACS Nano 8, 834 (2014).

[24] P. Yeh, A. Yariv, and C.-S. Hong, J. Opt. Soc. Am. 67, 423 (1977).

[25] S. Maier, Plasmonics: Fundamentals and Applications (Springer Science + Business Media, New York, 2007).

[26] E. Descrovi, P. Rivolo, L. Boarino, N. D. Leo, and F. Giorgis, in Organic and Hybrid Photonic Crystals (Springer Science + Business Media, New York, 2015), pp. 321-337.

[27] J. D. Joannopoulos, S. G. Johnson, J. N. Winn, and R. D. Meade, Photonic Crystals: Molding the Flow of Light (Princeton University Press, Princeton, NJ, 2011).

[28] E. D. Palik, Handbook of Optical Constants of Solids (Elsevier BV, Amsterdam, 1985).

[29] S. Y. Kim, Appl. Opt. 35, 6703 (1996).

[30] A. Sinibaldi, A. Fieramosca, R. Rizzo, A. Anopchenko, N. Danz, P. Munzert, C. Magistris, C. Barolo, and F. Michelotti, Opt. Lett. 39, 2947 (2014).

[31] A. Andryieuski, A. V. Lavrinenko, and S. V. Zhukovsky, Nanotechnology 26, 184001 (2015).

[32] See Supplemental Material at http://link.aps.org/supplemental/ 10.1103/PhysRevB.95.241402 for 1DPC geometrical parameters, dispersion relations and local field profiles, along with absorbance and CD spectra for a reference Flavin Mononucleotide film.

[33] S. G. Johnson and J. D. Joannopoulos, Opt. Express 8, 173 (2001).

[34] D. L. Jaggard and X. Sun, J. Opt. Soc. Am. A 9, 804 (1992).

[35] Historically, $\mathrm{CD}$ is expressed as ellipticity in mdeg with $\Delta A_{\text {mdeg }}=32980 \cdot \Delta A$. 